Revue d'histoire de l'Amérique française

RAS REVUE D.HISTOIRE DE L'AMÉRIQUE FRANÇAISE

\title{
Prudent-L. Mercure a-t-il écrit l'histoire du Madawaska ?
}

\section{J.-A. Robert Pichette}

Volume 8, numéro 2, septembre 1954

URI : https://id.erudit.org/iderudit/301653ar

DOI : https://doi.org/10.7202/301653ar

Aller au sommaire du numéro

Éditeur(s)

Institut d'histoire de l'Amérique française

ISSN

0035-2357 (imprimé)

1492-1383 (numérique)

Découvrir la revue

Citer cet article

Pichette, J.-A. R. (1954). Prudent-L. Mercure a-t-il écrit l'histoire du Madawaska ? Revue d'histoire de l'Amérique française, 8(2), 254-257. https://doi.org/10.7202/301653ar d'utilisation que vous pouvez consulter en ligne.

https://apropos.erudit.org/fr/usagers/politique-dutilisation/ 


\section{PRUDENT-L. MERCURE A-T-IL ÉCRIT L'HISTOIRE DU MADAWASKA?}

Le 11 mai 1753, naissait à l'Ile Saint-Jean, Louis Mercure, l'un des premiers colonisateurs du Madawaska. Le deuxième centenaire de cette naissance ne doit pas passer inaperçu, même s'il est quelque peu tard pour le rappeler!

Les descendants de Louis Mercure ne sont pas nombreux de nos jours. Cependant ils comptent parmi leurs ancêtres des hommes qui, à coup sûr, ont servi brillamment le pays. Dois-je citer Joseph Mercure, capitaine d'infanterie à l'Ile Saint-Jean et père de Louis Mercure? Dois-je citer aussi les noms de François Mercure marié le 31 janvier 1707 à Marie Perrot, fille de Joseph Perrot, seigneur d'Argentenay; et enfin Nazaire Mercure, premier colonisateur de la paroisse Saint-Jacques de Madawaska? Ne sont-ce pas Michel II et Pierre Mercure qui sont dits dans le "New-Brunswick Almanac" de 1838, enseignes militaires au Madawaska? Ces hommes ont, en quelque sorte, fait l'Histoire du Madawaska sans se douter qu'un de leurs descendants écrirait un jour cette Histoire de leur pays neuf. Cet homme, c'est Prudent Mercure, ancien archiviste à Ottawa.

Prudent-L. Mercure était un savant chercheur, un savant, un érudit. Ce qu'il a amassé de documents dans sa vie forme aujourd'hui trois vastes classeurs.

Ces documents sont inestimables pour un historien. Inestimables, surtout pour les Madawaskayens. Car c'est à l'Histoire de son pays natal que Prudent Mercure s'est attaché. Pourquoi cela? Personne d'autre ne l'avait fait avant lui. Le champ était vaste et libre! Mais pas pour longtemps cependant.

Ceux qui avaient écrit sur le Madawaska étaient rares. L'on peut citer le Rév. W. O. Raymond et le Père Cou ët ainsi que Ganong. C'est tout ou à peu près. D'ailleurs ces auteurs n'avaient fait qu'effleu- 
rer notre Histoire. Prudent Mercure se mit à l'œuvre et y mit toute sa vie. Car Prudent Mercure a écrit l'Histoire du Madawaska!

Aujourd'hui l'on considère le Sénateur Patrice Therriault et l'abbé Thomas Albert comme les seuls historiens du Madawaska. L'on se trompe, et de beaucoup! Mais les Madawaskayens, et plus particulièrement le clergé, se sont fait une idole du second de ces historiens, sans se préoccuper de celui qui avait fait le socle de cette statue.

Pourquoi n'a-t-on pas songé à donner à Prudent-L. Mercure la place qui lui convenait, c'est-à-dire la première place? Certes, l'abbé Thomas Albert a bien mérité du Madawaska, et bien naïf serait celui qui voudrait lui disputer cette gloire. Dans la préface de son "Histoire du Madawaska" l'abbé Albert a dit lui-même ce qui l'avait poussé à écrire l'Histoire de son petit coin de terre. Inutile d'y revenir.

Quel a donc été l'œuvre de l'abbé Albert? Je réponds immédiatement: "celui d'un enjoliveur de manuscrit"! Reportons-nous au Révérend Père Thomas Charland, o.p. qui écrivait:

Le véritable historien travaille sur les documents euxmêmes. Les bibliothèques regorgent de livres d'Histoire écrits par des auteurs qui n'ont fait que piller les travaux documentaires patiemment exécutés par d'autres, et qui se sont contentés de parer ces compilations de toutes les grâces du style, quand ils ne sont pas allés jusqu'à suppléer à l'absence ou à la pauvreté des sources par les efforts de leur imagination ou de leur psychologie de romanciers'

Ce jugement, à coup sûr, est sévère mais juste! L'abbé Albert s'est servi des documents de Prudent Mercure pour écrire l'Histoire du Madawaska. Il le dit lui-même. Il s'est contenté, cependant, de parer de toutes les grâces du style, cette compilation énorme qui n'était pas la sienne, pas plus que celle du Sénateur Patrice Therriault.

J'extrais de la correspondance de Mercure, une lettre de monsieur l'abbé Babineau, premier curé de Saint-Jacques du Mada-

1. Revue d'Histoire de l'Amerique franf̧aise, Volume 1 (No 3): 323. 
waska et datée du 8 janvier 1913. Voici ce que l'abbé Babineau écrivait à l'archiviste:

Cher ami,

J'ai reşu avec plaisir, et lu avec intérêt votre communication du 20 décembre dernier. Je suis heureux d'apprendre qu'enfin votre travail, que j'appelle très considerable et très important va être livré au public. Je vous en félicite d'avance tant je suis certain que c'est bien...

Ainsi donc au mois de janvier 1913, Prudent Mercure se préparait à publier un ouvrage qui visiblement concernait le Madawaska. Ne s'agirait-il pas de l'Histoire du Madawaska? Pourquoi pas?

Prudent Mercure écrivit aussi une réfutation d'un article du Père Couët intitule "La Fusion Des Deux Races". Mercure mettait sur la première ligne cette phrase latine:

\section{"SIC VOS NON VOBIS"!}

Or cette réfutation nous apprend que le $\mathrm{P}$. Couët avait écrit un article sur le Madawaska dans la revue de "La Nouvelle-France" durant les mois de septembre, octobre et novembre.

Prudent Mercure, après avoir lu ces articles, se demandait si ce travail était fondé sur des documents originaux, ou si c'était tout simplement une compilation d'un ouvrage historique quelconque. Mais il appert que le P. Couët avait écrit un article dans la revue de l'Action Sociale de Québec (1911). Cet article était daté du PetitSault, ancien nom de la cité d'Edmundston. Prudent Mercure dans sa réfutation nous dit ce qui suit:

“...il y avait aussi de passage à Saint-Hilaire de Madawaska à l'occasion de la dernière célébration de l'Assomption, plusieurs copieux manuscrits, y compris l'histoire manuscrite de cette région".

Et Mercure nous réfère à la revue "L'ASSOMPTION" du mois d'octobre de cette même année. Malheureusement le manuscrit n'est pas daté, car il s'agit d'une copie faite probablement par le sénateur Therriault.

Plus loin il ajoute: “... cette tranche d'Histoire d'une rare saveur, est tout simplement une compilation d'une partie de mon Histoire manuscrite de Madawaska". La chose est donc claire. Prudent Mercure a écrit l'Histoire du Madawaska et quelques auteurs 
se sont permis d'en copier des tranches, tout comme Emile Lauvrière, dans La Tragédie d'un Peuple, copiera l'Histoire du Madawaska de l'abbé Thomas Albert.

Il y a mieux! Prudent-L. Mercure, pour prouver ce qu'il avance, divise son manuscrit en deux colonnes. Celle de droite reproduit quelques chapitres de son Histoire, tandis que celle de gauche reproduit l'article du Père Couët. Il ne m'appartient pas de critiquer les articles du Père Couët. Cependant, le bon Père rencontra, ce jour là, un lutteur à son pied.

Prudent Mercure a même publié une partie de son Histoire dans le journal local, le 6 septembre 1905. Ce qui est une preuve supplémentaire en faveur de Mercure. Pour terminer cet article, je cite encore Prudent Mercure qui conclut ainsi sa réfutation du Père Couët:

...nous ne saurions ici contester le droit d'un auteur de reproduire certaine partie d'une étude déjà publiée pourvu que le lieu de provenance et l'auteur y soient indiqués. Cette règle de la plus stricte probité ne s'applique-t-elle pas avec autant de droit aux manuscrits inédits? Le même auteur nous dit à la page 515, numéro du mois de novembre, qu'il a l'intention de faire l'Histoire de l'Instruction publique du Madawaska. Nous ne savons ce que sera cette seconde compilation, mais nous tenons pour le moment à informer "La Nouvelle-France" que si cetté étude projetée est de la même provenance que "La fusion des deux races" nous prendrons les moyens que la loi met à notre disposition pour recouvrer les dommages-intérets causés par la publication de cette étude.

Prudent L. Mercure.

Voilà qui est évident! Prudent Mercure a bel et bien écrit l'Histoire du Madawaska. Il est notre premier historien et il est aussi le seul. Car ceux qui se sont basés sur L'Histoire du Madawaska de l'abbé Thomas Albert, pour écrire leurs "Histoires", n'ont tout simplement fait que copier Prudent Mercure. Puisse-t-il jouir enfin de la place qui est la sienne et qu'il a sûrement méritée.

J.-A. Robert Pichette, directeur de la Societé Historique du Madawaska. 